\title{
SRA-free condition by Zolotov for self-contracted curves and nondegeneracy of zz-distance for Möbius structures on the circle
}

\author{
Sergei Buyalo*
}

\begin{abstract}
SRA-free condition for metric spaces (that is, spaces without Small Rough Angles) was introduced by Zolotov to study rectifiability of selfcontracted curves in various metric spaces. We give a Möbius invariant version of this notion which allows to show that zz-distance associated with a respective Möbius structure on the circle is nondegenerate. This result is an important part of a solution to the inverse problem of Möbius geometry on the circle.
\end{abstract}

Keywords: Möbius structures, cross-ratio, harmonic 4-tuples, self-contracted curves

Mathematics Subject Classification: 51B10

\section{Introduction}

A Möbius structure $M$ on a set $X$ is a class of Möbius equivalent semimetrics on $X$, where two semi-metrics are equivalent if and only if they have the same cross-ratios on every 4 -tuple of points in $X$. A Möbius structure usually lives on the boundary at infinity of a hyperbolic space.

The inverse problem of Möbius geometry asks to describe Möbius structures which are induced by hyperbolic spaces. We are interested in the inverse problem for Möbius structures on the circle $X=S^{1}$. A natural condition for a such structure $M$ is formulated in [Bu18] as monotonicity axiom

(M) given a 4-tuple $q=(x, y, z, u) \in X^{4}$ such that the pairs $(x, y),(z, u)$ separate each other, we have

$$
|x y| \cdot|z u|>\max \{|x z| \cdot|y u|,|x u| \cdot|y z|\}
$$

for some and hence any semi-metric from $M$.

\footnotetext{
${ }^{*}$ Supported by RFFI Grant 17-01-00128a
} 
If we take one of the points of $q=(x, y, z, u)$, for example $u$, as infinitely remote for a semi-metric $|\cdot, \cdot|_{u} \in M$, then mononicity axiom tells us that

$$
|x y|_{u}>\max \left\{|x z|_{u},|y z|_{u}\right\},
$$

where $z$ is between $x, y$ on $X_{u}=X \backslash\{u\}=\mathbb{R}$. In particular, if a segment $x z \subset X_{u}$ is contained in a segment $x y \subset X_{u}$, then $|x z|_{u}<|x y|_{u}$. Surprisingly, exactly this condition defines self-contracted curves in metric spaces. Self-contracted curves usually appear as gradient curves of convex functions, and they play an important role in a number of questions. Basic problem for a self-contrated curve is to establish its rectifiability, see [DDDL, DDDR, [DLS], [LOZ], [Le], Ohta], [ST], Zo18].

We consider the set $\mathrm{Hm}$ of unordered harmonic pairs of unordered pairs of points in $X$ as a required filling of a Möbius structure $M$ in the inverse problem of Möbius geometry. Axiom (M) allows to uniquely define a line $\mathrm{h}_{a} \subset \mathrm{Hm}$ as the family of harmonic 4-tuples with a common axis, which is a pair of distinct points $a=(x, y)$ on $X$. Every harmonic pair $q=(a, b) \in \mathrm{Hm}$ has two axes. Thus moving along of a line, we have a possibility to change the axis of the line at any moment and move along the line determined by the other axis. This leads to the notion of zig-zag path. A zig-zag path, or zz-path, $S \subset \mathrm{Hm}$ is defined as finite sequence of segments $\sigma_{i}$ on lines in $\mathrm{Hm}$, where consecutive segments $\sigma_{i}, \sigma_{i+1}$ have a common end $q=\sigma_{i} \cap \sigma_{i+1} \in \mathrm{Hm}$ with axes determined by $\sigma_{i}, \sigma_{i+1}$.

We define a distance $\delta$ on $\mathrm{Hm}$ as

$$
\delta\left(q, q^{\prime}\right)=\inf _{S}|S|,
$$

where the infimum is taken over all zz-paths $S \subset \operatorname{Hm}$ from $q$ to $q^{\prime}$, and $|S|$ is the length of $S$ defined as the sum of the lengths of its sides. Its turns out that $\delta$ is finite and satisfies all the triangle axioms except maybe the positivity one. We replace axiom (M) by a slightly stonger axiom $(\mathrm{M}(\alpha))$.

$(\mathrm{M}(\alpha))$ Fix $0<\alpha<1$. Given a 4-tuple $q=(x, y, z, u) \in X^{4}$ such that the pairs $(x, y),(z, u)$ separate each other, we have

$$
|x y| \cdot|z u| \geq \max \{|x z| \cdot|y u|+\alpha|x u| \cdot|y z|, \alpha|x z| \cdot|y u|+|x u| \cdot|y z|\}
$$

for some and hence any semi-metric from $M$.

This is a Möbius invariant version of the SRA-free condition introduced by Zolotov in [Zo18].

Now, our main result is as follows.

Theorem 1.1. Let $M$ be a ptolemaic Möbius structure of the circle $X$ which satisfies axiom $(M(\alpha))$ for some $0<\alpha<1$, such that the $M$-topology is the topology of $S^{1}$. Then the distance $\delta$ on $\mathrm{Hm}$ is nondegenerate, $\delta\left(q, q^{\prime}\right) \neq 0$ if and only if $q \neq q^{\prime}$, the $\delta$-metric topology on $\mathrm{Hm}$ coincides with one induced from $X^{4}$, and the metric space $(\mathrm{Hm}, \delta)$ is complete. In particual, $(\mathrm{Hm}, \delta)$ is a proper geodesic metric space. 


\section{Möbius structures}

\subsection{Basic notions}

Let $X$ be a set. A 4-tuple $q=(x, y, z, u) \in X^{4}$ is said to be admissible if no entry occurs three or four times in $q$. A 4-tuple $q$ is nondegenerate, if all its entries are pairwise distinct. Let $\mathcal{P}_{4}=\mathcal{P}_{4}(X)$ be the set of all ordered admissible 4-tuples of $X, \operatorname{reg} \mathcal{P}_{4} \subset \mathcal{P}_{4}$ the set of nondegenerate 4-tuples.

A function $d: X^{2} \rightarrow \widehat{\mathbb{R}}=\mathbb{R} \cup\{\infty\}$ is said to be a semi-metric, if it is symmetric, $d(x, y)=d(y, x)$ for each $x, y \in X$, positive outside the diagonal, vanishes on the diagonal and there is at most one infinitely remote point $\omega \in X$ for $d$, i.e. such that $d(x, \omega)=\infty$ for some $x \in X \backslash\{\omega\}$. Moreover, we require that if $\omega \in X$ is such a point, then $d(x, \omega)=\infty$ for all $x \in X$, $x \neq \omega$. A metric is a semi-metric that satisfies the triangle inequality.

A Möbius structure $M$ on $X$ is a class of Möbius equivalent semi-metrics on $X$, where two semi-metrics are equivalent if and only if they have the same cross-ratios on every $q \in \operatorname{reg} \mathcal{P}_{4}$.

Given $\omega \in X$, there is a semi-metric $d_{\omega} \in M$ with infinitely remote point $\omega$. It can be obtained from any semi-metric $d \in M$ for which $\omega$ is not infinitely remote by a metric inversion,

$$
d_{\omega}(x, y)=\frac{d(x, y)}{d(x, \omega) d(y, \omega)} .
$$

Such a semi-metric is unique up to a homothety, see [FS13], and we use notation $|x y|_{\omega}=d_{\omega}(x, y)$ for the distance between $x, y \in X$ in that semimetric. We also use notation $X_{\omega}=X \backslash\{\omega\}$.

Every Möbius structure $M$ on $X$ determines the $M$-topology whose subbase is given by all open balls centered at finite points of all semi-metrics from $M$ having infinitely remote points.

Example 2.1. Our basic example is the canonical Möbius structure $M_{0}$ on the circle $X=S^{1}$. We think of $S^{1}$ as the unit circle in the plane, $S^{1}=\left\{(x, y) \in \mathbb{R}^{2}: x^{2}+y^{2}=1\right\}$. For $\omega=(0,1) \in X$ the stereographic projection $X_{\omega} \rightarrow \mathbb{R}$ identifies $X_{\omega}$ with real numbers $\mathbb{R}$. We let $d_{\omega}$ be the standard metric on $\mathbb{R}$, that is, $d_{\omega}(x, y)=|x-y|$ for any $x, y \in \mathbb{R}$. This generates a Möbius structure on $X$ which is called canonical. The basic feature of the canonical Möbius structure on $X=S^{1}$ is that for any 4-tuple $(\sigma, x, y, z) \subset X$ with the cyclic order $\sigma x y z$ we have $d_{\sigma}(x, y)+d_{\sigma}(y, z)=$ $d_{\sigma}(x, z)$.

\subsection{Harmonic pairs}

From now on, we assume that $X$ is the circle, $X=S^{1}$. It is convenient to use unordered pairs $(x, y) \sim(y, x)$ of distinct points on $X$, and we denote 
their set by aY $=S^{1} \times S^{1} \backslash \Delta / \sim$, where $\Delta=\left\{(x, x): x \in S^{1}\right\}$ is the diagonal. A pair $q=(a, b) \in \mathrm{aY} \times \mathrm{aY}$ is harmonic if

$$
|x z| \cdot|y u|=|x u| \cdot|y z|
$$

for some and hence any semi-metric of the Möbius structure, where $a=$ $(x, y), b=(z, u)$. The pair $a$ is called the left axis of $q$, while $b$ the right axis. We denote by Harm the set of harmonic pairs, Harm $\subset$ aY $\times$ aY, of the given Möbius structure. There is a canonical involution $j:$ Harm $\rightarrow$ Harm without fixed points given by $j(a, b)=(b, a)$. Note that $j$ permutes left and right axes. The quotient space we denote by $\mathrm{Hm}:=$ Harm $/ j$. In other words, $\mathrm{Hm}$ is the set of unordered harmonic pairs of unordered pairs of points in $X$.

\section{$2.3 \quad$ Axioms}

We list a set of axioms for a Möbius structure $M$ on the circle $X=S^{1}$.

(T) Topology: $M$-topology on $X$ is that of $S^{1}$.

$(\mathrm{M}(\alpha))$ Monotonicity: Fix $0<\alpha<1$. Given a 4-tuple $q=(x, y, z, u) \in X^{4}$ such that the pairs $(x, y),(z, u)$ separate each other, we have

$$
|x y| \cdot|z u| \geq \max \{|x z| \cdot|y u|+\alpha|x u| \cdot|y z|, \alpha|x z| \cdot|y u|+|x u| \cdot|y z|\}
$$

for some and hence any semi-metric from $M$.

(P) Ptolemy: for every 4-tuple $q=(x, y, z, u) \in X^{4}$ we have

$$
|x y| \cdot|z u| \leq|x z| \cdot|y u|+|x u| \cdot|y z|
$$

for some and hence any semi-metric from $M$.

A Möbius structure $M$ on the circle $X$ that satisfies axioms T, $\mathrm{M}(\alpha), \mathrm{P}$ is said to be strictly monotone.

Remark 2.2. Axiom $\mathrm{M}(\alpha)$ is motivated by the work [Zo18] of V. Zolotov.

Remark 2.3. Axiom $\mathrm{P}$ is satisfied, for example, for the Möbius structure on the boundary at infinity of any CAT $(-1)$ space, see [FS12].

Remark 2.4. The canonical Möbius structure $M_{0}$ on $X=S^{1}$ clearly satisfies Axioms $\mathrm{T}, \mathrm{M}(\alpha), \mathrm{P}$.

We derive some immediate corollaries from the axioms. It follows from $\mathrm{P}$ that any semi-metric from $M$ with an infinitely remote point is a metric, i.e. it satisfies the triange inequality.

Corollary 2.5. For every $\omega \in X$ the metric space $X_{\omega}$ is complete. 
Proof. By Axiom (T), $X_{\omega}$ is homeomorphic to $\mathbb{R}$. Since $\omega$ is infinitely remote, the space $X_{\omega}$ is unbounded in the respective metric. These two properties together easily imply that $X_{\omega}$ is complete.

A choice of $\omega \in X$ uniquely determines the interval $x y \subset X_{\omega}$ for any distinct $x, y \in X$ different from $\omega$ as the arc in $X$ with the end points $x, y$ that does not contain $\omega$. As an useful implication of Axiom $\mathrm{M}(\alpha)$ we have

Corollary 2.6. Axiom $M(\alpha)$ implies the following. Assume for a nondegenerate 4-tuple $q=(x, y, z, u) \in \operatorname{reg} \mathcal{P}_{4}$ the interval $x z \subset X_{u}$ is contained in $x y, x z \subset x y \subset X_{u}$. Then $|x z|_{u}<|x y|_{u}$. That is, $X_{u}$ is self-contraced for any $u \in X$.

Proof. By the assumption, the pairs $(x, y),(z, u)$ separate each other. Hence, by Axiom $\mathrm{M}(\alpha)$ we have $\left.|x z|_{u}|<| x y\right|_{u}$.

Corollary 2.7. For any harmonic pair $((x, y),(z, u)) \in$ Harm the pairs $(x, y),(z, u) \in$ aY separate each other.

Proof. In the metric from $M$ with infinitely remote point $u$ we have $|x z|_{u}=$ $|z y|_{u}$. By Corollary 2.6, $z$ lies between $x, y$ on the line $X_{u}$. Hence, the pairs $(x, y),(z, u)$ separate each other.

\section{$3 \quad$ Lines and zigzag paths}

Here we briefly recall definitions and some properties of lines and zigzag paths from Bu18.

\subsection{Lines}

Lemma 3.1. Given $a \in$ aY and $x \in X, x \notin a$, there is a uniquely determined $y \in X$ such that the pair $(a, b)$ is harmonic, $(a, b) \in \mathrm{Hm}$, where $b=(x, y)$.

Proof. Let $a=(z, u)$. We take a metric from $M$ with infinitely remote point $u$. The distance function $y \mapsto|z y|_{u}$ is continuous on $X_{u}$ (see [Bu17, Lemma 4.1]), thus there is $y \in X_{u}$ with $|x z|_{u}=|z y|_{u}$. Hence the pair $(a, b)$, where $b=(x, y)$ is harmonic. By Corollary 2.6, the point $y$ is uniquely determined.

We denote by $\rho_{a}(x)=y$ the point $y$ from Lemma 3.1. The line with axis $a \in \mathrm{aY}$ is defined as the set $\mathrm{h}_{a} \subset \mathrm{Hm}$ which consists of all pairs $q=(a, b)$ with $b=\left(x, \rho_{a}(x)\right)$ where $x$ run over an arc in $X$ determined by $a$. This is well defined because $\rho_{a}: X \rightarrow X$ is involutive, $\rho_{a}^{2}=\mathrm{id}$ (we extend $\rho_{a}$ to $a=(z, u)$ by $\left.\rho_{a}(z)=z, \rho_{a}(u)=u\right)$. In this case, we use notation $x_{a}:=b$ and say that $x_{a} \in \mathrm{h}_{a}$ is the projection of $x$ to the line $\mathrm{h}_{a}$. 
For more about lines see Bu18. In particual, every line is homeomorphic to the real line $\mathbb{R}$, different points on a line are in strong causal relation, that is, either of them lies on an open $\operatorname{arc}$ in $X$ determined by the other one, and vice versa, given $b, b^{\prime} \in$ aY in strong causal relation, there exists a unique line $\mathrm{h}_{a}$ through $b, b^{\prime}$, see [Bu18, Lemma 3.2, Lemma 4.2]. In this case, the pair $a \in \mathrm{aY}$ (or the line $\mathrm{h}_{a}$ ) is called the common perpendicular to $b, b^{\prime}$.

The segment $q q^{\prime}$ of a line $\mathrm{h}_{a}$ with $q=(a, b), q^{\prime}=\left(a, b^{\prime}\right) \in \mathrm{h}_{a}$ is defined as the union of $q, q^{\prime}$ and all $q^{\prime \prime}=\left(a, b^{\prime \prime}\right) \in \mathrm{h}_{a}$ such that $b^{\prime \prime}$ separates $b, b^{\prime}$. The last means that $b$ and $b^{\prime}$ lie on different open $\operatorname{arcs}$ in $X$ determined by $b^{\prime \prime}$. The points $q, q^{\prime}$ are the ends of $q q^{\prime}$. The segment $q q^{\prime} \subset \mathrm{h}_{a}$ is homeomorphic to the standard segment $[0,1]$.

\subsection{Distance between harmonic pairs with common axis}

Given two harmonic pairs $q, q^{\prime} \in \mathrm{Hm}$ with a common axis, say $q=(a, b)$ and $q^{\prime}=\left(a, b^{\prime}\right)$, we define the distance $\left|q q^{\prime}\right|$ between them as

$$
\left|q q^{\prime}\right|=\left|\ln \frac{\left|x z^{\prime}\right| \cdot|y z|}{|x z| \cdot\left|y z^{\prime}\right|}\right|
$$

for some and hence any semi-metric on $X$ from $M$, where $a=(x, y), b=$ $(z, u), b^{\prime}=\left(z^{\prime}, u^{\prime}\right) \in \mathrm{aY}$.

One easily checks that every line $\mathrm{h}_{a} \subset \mathrm{Hm}$ with this distance is isometric to the real line $\mathbb{R}$ with the standard distance.

\subsection{Width of a strip}

We say that a 4-tuple $p=(a, b) \in X^{4}$ with $a=(x, y), b=(u, z)$ is a strip if $a, b$ are in the strong causal relation and the pairs $(x, z),(u, y)$ separate each other. Note that $p^{\prime}=(b, c) \in X^{4}$ with $b=(x, u), c=(y, z)$ is also a strip based on the same 4-tuple $(x, y, u, z) \in X^{4}$.

Since the pairs $a, b$ are in the strong causal relation, there is uniquely determined common perpendicular $s=(v, w)$ to $a, b$. We assume that $w$ lies on the arc in $X$ determined by $b$ which does not contain $a$. We denote $g=|v x|_{w}, h=|v u|_{w}$. Then $|v y|_{w}=g,|v z|_{w}=h$ because $s$ is the common perpendicular to $a, b$, and $h>g$ by mononicity and the choice of the infinitely remote point $w$. We use notation $p=(a, b, s)$ for a strip with common perpendicular $s$. Note that $s$ is uniquely determined by $(a, b)$, and we add $s$ to fix notation. A strip $p=(a, b, s)$ is said to be narrow if $|x u|_{w}$, $|y z|_{w} \leq g=|v x|_{w}=|v y|_{w}$.

We define the width of the strip $p$ as the length $l=\operatorname{width}(p)$ of the segment $x_{s} u_{s}=y_{s} z_{s} \subset \mathrm{h}_{s}$ on the line $\mathrm{h}_{s}$.

Lemma 3.2. For any strip $p=(a, b, s)$ we have

$$
\operatorname{width}(p) \leq 2 \sqrt{\frac{|x u||y z|}{|x y||z u|}},
$$


where $a=(x, y), b=(u, z)$.

Lemma 3.3. Let $p=(a, b, s)$ be a strip with $a=(x, y), b=(u, z), s=$ $(v, w)$. Then for the width $l=\operatorname{width}(p)$ of $p$ we have

$$
2 \sinh (l / 2) \geq \alpha(1+\alpha) \sqrt{\frac{|x u||y z|}{|x y||u z|}}
$$

and

$$
|x u|_{w},|y z|_{w} \leq \frac{e^{l}-1}{\alpha(1+\alpha)}|x y|_{w}, \quad \alpha \leq \frac{|x u|_{w}}{|y z|_{w}} \leq \frac{1}{\alpha},
$$

where $\alpha$ is the constant from Axiom $M(\alpha)$.

Proof of Lemmas 3.2 and 3.3. In the metric ||$_{w}$ on $X_{w}$ with infinitely remote point $w$ we have $|x u|_{w},|y z|_{w} \geq h-g$ and also $|x y|_{w} \leq 2 g,|u z|_{w} \leq 2 h$ by the triange inequality. Thus

$$
\frac{|x u|_{w}|y z|_{w}}{|x y|_{w}|u z|_{w}} \geq \frac{(h-g)^{2}}{4 g h}=\sinh ^{2}(l / 2) \geq(l / 2)^{2},
$$

where $l=\operatorname{width}(p)$, because $e^{l}=h / g$, see Eq. (3). This proves Lemma 3.2,

The pairs $(v, z)$ and $(w, y)$ separate each other. Thus by Axiom $\operatorname{M}(\alpha)$ we have

$$
|v z||w y| \geq \max \{|y z||v w|+\alpha|z w||v y|, \alpha|y z||v w|+|z w||v y|\}
$$

in any semi-metric of the Möbius structure M. Taking the metric with infinitely remote point $w$, we obtain

$$
h \geq \max \left\{|y z|_{w}+\alpha g, \alpha|y z|_{w}+g\right\} .
$$

The inequality (4) implies $\alpha|y z|_{w} \leq h-g$. Similarly $\alpha|u x|_{w} \leq h-g$.

Again, by Axiom $\mathrm{M}(\alpha),|x y|_{w} \geq|x v|_{w}+\alpha|v y|_{w}=(1+\alpha) g$ and similarly $|u z|_{w} \geq(1+\alpha) h$. Thus

$$
\frac{|y z||x u|}{|x y||u z|} \leq \frac{(h-g)^{2}}{\alpha^{2}(1+\alpha)^{2} g h} .
$$

Since $e^{l}=h / g$, this gives

$$
4(\sinh l / 2)^{2} \geq \alpha^{2}(1+\alpha)^{2} \frac{|y z||x u|}{|x y||u z|},
$$

hence the first estimate of Lemma 3.3

Estimates above also give $|u x|_{w} \leq \frac{1}{\alpha}(h-g)=\frac{e^{l}-1}{\alpha} g=\frac{e^{l}-1}{\alpha(1+\alpha)}|x y|_{w}$ and similarly $|y z|_{w} \leq \frac{e^{l}-1}{\alpha(1+\alpha)}|x y|_{w}$. Using the inequalities $|y z|_{w},|x u|_{w} \geq$ $h-g$ together with the previous estimates from above, we obtain the second estimate of Lemma 3.3 . 


\subsection{Zigzag paths}

Every harmonic pair $q=(a, b) \in \mathrm{Hm}$ has two axes. Thus moving along of a line, we have a possibility to change the axis of the line at any moment and move along the line determined by the other axis. This leads to the notion of zig-zag path. A zig-zag path, or zz-path, $S \subset \mathrm{Hm}$ is defined as finite (maybe empty) sequence of segments $\sigma_{i}$ in $\mathrm{Hm}$, where consecutive segments $\sigma_{i}, \sigma_{i+1}$ have a common end $q=\sigma_{i} \cap \sigma_{i+1} \in \mathrm{Hm}$ with axes determined by $\sigma_{i}, \sigma_{i+1}$. Segments $\sigma_{i}$ are also called sides of $S$, while a vertex of $S$ is an end of a side. Given $q, q^{\prime} \in \mathrm{Hm}$, there is a zz-path $S$ in Hm with at most five sides that connects $q$ and $q^{\prime}$ (see [Bu18, Lemma 3.3]).

\section{Metric on $\mathrm{Hm}$}

\subsection{Distance $\delta$ on $\mathrm{Hm}$}

Let $S=\left\{\sigma_{i}\right\}$ be a zz-path in Hm. We define the length of $S$ as the sum $|S|=\sum_{i}\left|\sigma_{i}\right|$ of the length of its sides. Now, we define a distance $\delta$ on $\mathrm{Hm}$ by

$$
\delta\left(q, q^{\prime}\right)=\inf _{S}|S|,
$$

where the infimum is taken over all zz-paths $S \subset \operatorname{Hm}$ from $q$ to $q^{\prime}$.

One easily sees that $\delta$ is a finite pseudometric on $\mathrm{Hm}$, see [Bu18, Proposition 6.2]. Now, we show that $\delta$ is a metric.

Lemma 4.1. Fix a harmonic $q \in \mathrm{Hm}, q=(a, s)$ with $a=(x, y), s=$ $(v, w) \in \mathrm{aY}$. Let $p^{\prime}=\left(a^{\prime}, b^{\prime}, s^{\prime}\right)$ be a strip with $a^{\prime}=\left(x^{\prime}, y^{\prime}\right), b^{\prime}=\left(u^{\prime}, z^{\prime}\right)$, $s^{\prime}=\left(v^{\prime}, w^{\prime}\right)$ such that $w$ lies on the arc in $X$ determined by $b^{\prime}$ that contains $w^{\prime}$. Then

$$
\alpha \beta \leq \frac{\left|x^{\prime} u^{\prime}\right|_{w}}{\left|y^{\prime} z^{\prime}\right|_{w}} \leq \frac{1}{\alpha \beta}
$$

for some $\beta=\beta\left(p^{\prime}, w\right)<1$. If, in addition $a^{\prime}, b^{\prime} \in U_{\varepsilon}(a), s^{\prime} \in V_{\varepsilon}(s)$ for a sufficiently small $\varepsilon, 0<\varepsilon<1 / 8$, where $U_{\varepsilon}(a)=\left\{\left(x^{\prime}, y^{\prime}\right) \in\right.$ aY : $\left.\left|x x^{\prime}\right|_{w}<\varepsilon,\left|y y^{\prime}\right|_{w}<\varepsilon\right\}, V_{\varepsilon}(s)=\left\{\left(v^{\prime}, w^{\prime}\right) \in \mathrm{aY}:\left|v v^{\prime}\right|_{x}<\varepsilon,\left|w w^{\prime}\right|_{x}<\varepsilon\right\}$, then $\beta \geq 1-8 \varepsilon$. In this case,

$$
2 \sinh \left(l^{\prime} / 2\right) \geq c \max \left\{\left|x^{\prime} u^{\prime}\right|_{w},\left|y^{\prime} z^{\prime}\right|_{w}\right\}
$$

with $c=\frac{\alpha(1+\alpha) \sqrt{\alpha}}{2} \sqrt{1-8 \varepsilon}$, where $l^{\prime}=\operatorname{width}\left(p^{\prime}\right)$.

Proof. Using the metric inversion (11), we have

$$
\left|x^{\prime} u^{\prime}\right|_{w}=\frac{\left|x^{\prime} u^{\prime}\right|_{w^{\prime}}}{\left|x^{\prime} w\right|_{w^{\prime}}\left|u^{\prime} w\right|_{w^{\prime}}} \text { and } \quad\left|y^{\prime} z^{\prime}\right|_{w}=\frac{\left|y^{\prime} z^{\prime}\right|_{w^{\prime}}}{\left|y^{\prime} w\right|_{w^{\prime}}\left|z^{\prime} w\right|_{w^{\prime}}} .
$$

Thus

$$
\frac{\left|x^{\prime} u^{\prime}\right|_{w}}{\left|y^{\prime} z^{\prime}\right|_{w}}=\gamma \frac{\left|x^{\prime} u^{\prime}\right|_{w^{\prime}}}{\left|y^{\prime} z^{\prime}\right|_{w^{\prime}}},
$$


where

$$
\gamma=\frac{\left|y^{\prime} w\right|_{w^{\prime}}\left|z^{\prime} w\right|_{w^{\prime}}}{\left|x^{\prime} w\right|_{w^{\prime}}\left|u^{\prime} w\right|_{w^{\prime}}}
$$

By Lemma 3.3

$$
2 \sinh \left(l^{\prime} / 2\right) \geq \alpha(1+\alpha) \sqrt{\frac{\left|x^{\prime} u^{\prime}\right|\left|y^{\prime} z^{\prime}\right|}{\left|x^{\prime} y^{\prime}\right|\left|u^{\prime} z^{\prime}\right|}} \quad \text { and } \quad \alpha \leq \frac{\left|x^{\prime} u^{\prime}\right|_{w^{\prime}}}{\left|y^{\prime} z^{\prime}\right|_{w^{\prime}}} \leq \frac{1}{\alpha} .
$$

Using that $|x w|_{w^{\prime}}=1 /\left|x w^{\prime}\right|_{w}$, we obtain

$$
\gamma=\frac{\left|x^{\prime} w^{\prime}\right|_{w}\left|u^{\prime} w^{\prime}\right|_{w}}{\left|y^{\prime} w^{\prime}\right|_{w}\left|z^{\prime} w^{\prime}\right|_{w}}
$$

If $w^{\prime}=w$ then $\gamma=1$. Otherwise, consider two cases:

(1) $w$ lies on the arc in $X$ between $w^{\prime}$ and $z^{\prime}$ that does not contain $y^{\prime}$. In this case $u^{\prime} w^{\prime} \subset x^{\prime} w^{\prime}, y^{\prime} w^{\prime} \subset z^{\prime} w^{\prime}, x^{\prime} w^{\prime} \subset y^{\prime} w^{\prime}$ in $X_{w}$. Thus $\left|u^{\prime} w^{\prime}\right|_{w}<$ $\left|x^{\prime} w^{\prime}\right|_{w},\left|y^{\prime} w^{\prime}\right|_{w}<\left|z^{\prime} w^{\prime}\right|_{w},\left|x^{\prime} w^{\prime}\right|_{w}<\left|y^{\prime} w^{\prime}\right|_{w}$ and hence

$$
\gamma \leq \frac{\left|x^{\prime} w^{\prime}\right|_{w}^{2}}{\left|y^{\prime} w^{\prime}\right|_{w}^{2}}<1
$$

In this case, we put $\beta=\gamma$ and obtain

$$
\alpha \beta \leq \frac{\left|x^{\prime} u^{\prime}\right|_{w}}{\left|y^{\prime} z^{\prime}\right|_{w}} \leq \frac{1}{\alpha \beta}
$$

because $\gamma<1 / \gamma$.

(2) $w$ lies on the arc in $X$ between $w^{\prime}$ and $u^{\prime}$ that does not contain $x^{\prime}$. In this case $x^{\prime} w^{\prime} \subset u^{\prime} w^{\prime}, z^{\prime} w^{\prime} \subset y^{\prime} w^{\prime}, z^{\prime} w^{\prime} \subset x^{\prime} w^{\prime}$ in $X_{w}$. Thus $\left|u^{\prime} w^{\prime}\right|_{w}>$ $\left|x^{\prime} w^{\prime}\right|_{w},\left|z^{\prime} w^{\prime}\right|_{w}<\left|y^{\prime} w^{\prime}\right|_{w},\left|x^{\prime} w^{\prime}\right|_{w}>\left|y^{\prime} w^{\prime}\right|_{w}$ and hence

$$
\gamma \geq \frac{\left|x^{\prime} w^{\prime}\right|_{w}^{2}}{\left|y^{\prime} w^{\prime}\right|_{w}^{2}}>1
$$

In this case, we put $\beta=1 / \gamma$ and obtain

$$
\alpha \beta \leq \frac{\left|x^{\prime} u^{\prime}\right|_{w}}{\left|y^{\prime} z^{\prime}\right|_{w}} \leq \frac{1}{\alpha \beta}
$$

because $\gamma>1 / \gamma$.

Finally, the condition $s^{\prime} \in V_{\varepsilon}(s)$ implies that $\left|w^{\prime} w\right|_{x}<\varepsilon$ and hence $\left|w^{\prime} x\right|_{w}>1 / \varepsilon$ by the metric inversion (10). We put $t:=\left|w^{\prime} x\right|_{w}$.

The condition $a^{\prime}, b^{\prime} \in U_{\varepsilon}(a)$ implies

$$
t-\varepsilon \leq\left|w^{\prime} x^{\prime}\right|_{w},\left|w^{\prime} u^{\prime}\right|_{w} \leq t+\varepsilon
$$

and

$$
\left|w^{\prime} y\right|_{w}-\varepsilon \leq\left|w^{\prime} y^{\prime}\right|_{w},\left|w^{\prime} z^{\prime}\right|_{w} \leq\left|w^{\prime} y\right|_{w}+\varepsilon
$$


by the triange inequality.

We can assume without loss of generality that $|x y|_{w}=1$. Since $w^{\prime}$ lies on the $\operatorname{arc}$ in $X$ determined by $a$ that contains $w$, we have $t-1 \leq\left|w^{\prime} y\right|_{w} \leq t+1$ and hence $t-1-\varepsilon \leq\left|w^{\prime} y^{\prime}\right|_{w} \leq t+1+\varepsilon$. Using that $t>1 / \varepsilon$ we obtain

$$
\gamma \geq \frac{(t-\varepsilon)^{2}}{(t+1+\varepsilon)^{2}} \geq \frac{\left(1-\varepsilon^{2}\right)^{2}}{\left(1+\varepsilon+\varepsilon^{2}\right)^{2}} \geq(1-2 \varepsilon)^{2} \geq 1-8 \varepsilon
$$

and similarly

$$
\gamma \leq \frac{(t+\varepsilon)^{2}}{(t-1-\varepsilon)^{2}} \leq \frac{\left(1+\varepsilon^{2}\right)^{2}}{\left(1-\varepsilon-\varepsilon^{2}\right)^{2}} \leq(1+2 \varepsilon)^{2} \leq 1+8 \varepsilon .
$$

For the first case, $\beta=\gamma$, we take the first estimate above and obtain $\beta \geq$ $1-8 \varepsilon$. For the second case, $\beta=1 / \gamma$, we take the second estimate above and obtain $\beta \geq 1 /(1+8 \varepsilon) \geq 1-8 \varepsilon$.

The condition $a^{\prime}, b^{\prime} \in U_{\varepsilon}(a)$ also implies $\left|x^{\prime} y^{\prime}\right|_{w},\left|u^{\prime} z^{\prime}\right|_{w} \leq 1+2 \varepsilon$. Using (5), we obtain

$$
2 \sinh \left(l^{\prime} / 2\right) \geq c \max \left\{\left|x^{\prime} u^{\prime}\right|_{w},\left|y^{\prime} z^{\prime}\right|_{w}\right\}
$$

with $c=\frac{\alpha(1+\alpha) \sqrt{\alpha}}{2} \sqrt{1-8 \varepsilon}$.

Lemma 4.2. Given $\varepsilon, 0<\varepsilon \leq 1 / 16$, there is $t=t(\varepsilon)>0$ such that for every zz-path $S$ in $\mathrm{Hm}$ with $|S|<t$ and the initial vertex $q=q_{1}=(a, s)$, $a=(x, y), s=(v, w) \in$ aY, the vertices $q_{i}=\left(a_{i}, s_{i}\right), i \geq 1$, of $S$ lie in $U_{\varepsilon}(a) \times V_{\varepsilon}(s)$, where $U_{\varepsilon}(a)=\left\{\left(x^{\prime}, y^{\prime}\right) \in \mathrm{aY}:\left|x x^{\prime}\right|_{w}<\varepsilon,\left|y y^{\prime}\right|_{w}<\varepsilon\right\}$, $V_{\varepsilon}(s)=\left\{\left(v^{\prime}, w^{\prime}\right) \in \mathrm{aY}:\left|v v^{\prime}\right|_{x}<\varepsilon,\left|w w^{\prime}\right|_{x}<\varepsilon\right\}$, and

$$
\left|q_{i} q_{i+1}\right| \geq c \max \left\{\left|x_{i} x_{i+1}\right|_{w},\left|y_{i} y_{i+1}\right|_{w}\right\}
$$

for every odd $i \geq 1$, where $a_{i}=\left(x_{i}, y_{i}\right) \in \mathrm{aY}$,

$$
\left|q_{i} q_{i+1}\right| \geq c \max \left\{\left|v_{i} v_{i+1}\right|_{x},\left|w_{i} w_{i+1}\right|_{x}\right\}
$$

for every even $i \geq 2$, where $s_{i}=\left(v_{i}, w_{i}\right) \in \mathrm{aY}$, and some constant $c=$ $c(\alpha)>0$.

Proof. We put $c=\frac{\alpha(1+\alpha) \sqrt{\alpha}}{16}$ and take $t>0$ such that $\sinh (t / 2) \leq c \varepsilon$.

Denote $s_{i}=\left(v_{i}, w_{i}\right) \in$ aY, $i \geq 1, s_{1}=s$. Assume without loss of generality that the first side $\sigma_{1}=q_{1} q_{2}$ of $S$ lies on the line $\mathrm{h}_{s_{1}}$. Then we have $s_{i+1}=s_{i}$ for odd $i$, and $a_{i+1}=a_{i}$ for even $i$.

We argue by induction on the number $n$ of sides of $S$. The base of the induction: $n=1$.

We have $q_{1}=q=(a, s)$ with $a=(x, y), s=(v, w) \in \mathrm{aY}, q_{2}=\left(a_{2}, s\right)$ with $a_{2}=\left(x_{2}, y_{2}\right)$, and $s$ is the common perpendicular to the strip $p_{1}=$ $\left(a, a_{2}\right)$. The width of $p_{1}$ equals the length $l_{1}$ of the side $q_{1} q_{2},\left|q_{1} q_{2}\right|=l_{1}=$ $\operatorname{width}\left(p_{1}\right)$. We assume that $w$ lies on the $\operatorname{arc}$ in $X$ determined by $a_{2}$ that does 
not contain $a$, and that $p_{1}$ is narrow, that is, $\left|x x_{2}\right|_{w},\left|y y_{2}\right|_{w} \leq|v x|_{w}=|v y|_{w}$, see sect. 3.3. Note that $\left|v x_{2}\right|_{w} \geq|v x|_{w}+\alpha\left|x x_{2}\right|_{w}$ by Axion $\mathrm{M}(\alpha)$. Thus $e^{l_{1}}=\left|v x_{2}\right|_{w} /|v x|_{w} \geq 1+\alpha\left(\left|x x_{2}\right|_{w} /|v x|_{w}\right)$. Hence if $l_{1} \leq \ln (1+\alpha)$, then $\left|x x_{2}\right|_{w} \leq|v x|_{w}$ and similarly $\left|y y_{2}\right|_{w} \leq|v y|_{w}$, i.e., $p_{1}$ is narrow.

By Lemma 3.3 we have

$$
2 \sinh \left(l_{1} / 2\right) \geq \alpha(1+\alpha) \sqrt{\frac{\left|x x_{2}\right|\left|y y_{2}\right|}{|x y|\left|x_{2} y_{2}\right|}}
$$

and

$$
\alpha \leq \frac{\left|x x_{2}\right|_{w}}{\left|y y_{2}\right|_{w}} \leq \frac{1}{\alpha}
$$

Taking the normalization $|x y|_{w}=1$ for the metric of $X_{w}$, we obtain $\left|x_{2} y_{2}\right|_{w} \leq$ $1+\left|x x_{2}\right|_{w}+\left|y y_{2}\right|_{w} \leq 1+2|v x|_{w} \leq 2$ by the triange inequality. Hence $2 \sinh \left(l_{1} / 2\right) \geq \frac{\alpha(1+\alpha) \sqrt{\alpha}}{2}\left|x x_{2}\right|_{w}$. We see that

$$
\max \left\{\left|x x_{2}\right|_{w},\left|y y_{2}\right|_{w}\right\} \leq \frac{4}{\alpha(1+\alpha) \sqrt{\alpha}} \sinh \left(l_{1} / 2\right) \leq \varepsilon / 4
$$

for $|S|=l_{1}<t$. Thus $q_{i}$ lies in $U_{\varepsilon / 2}(a) \times\{s\} \subset U_{\varepsilon / 2}(a) \times V_{\varepsilon / 2}(s)$ for $i=1,2$, and

$$
l_{1}=\left|q_{1} q_{2}\right| \geq c \max \left\{\left|x_{1} x_{2}\right|_{w},\left|y_{1} y_{2}\right|_{w}\right\},
$$

because $t \leq 1 / 64$ by our assumptions $c \leq 1 / 8, \varepsilon \leq 1 / 16$, and thus $l \geq$ $\sinh (l / 2)$ for $l \leq t$.

Assume the lemma is proved for all $n<k$, and consider a zz-path $S$ with $k$ sides and $|S|<t$. By the inductive assumption, $q_{i} \in U_{\varepsilon / 2}(a) \times V_{\varepsilon / 2}(s)$ for $i=1, \ldots, k$. We have to show that the inclusion above holds also for $i=k+1$, and assuming that $k$ is odd, that

$$
\left|q_{k} q_{k+1}\right| \geq c \max \left\{\left|x_{k} x_{k+1}\right|_{w},\left|y_{k} y_{k+1}\right|_{w}\right\} .
$$

Let $p_{k}=\left(q_{k}, q_{k+1}\right)$ be the $k$ th strip with the common perpendicular $s_{k}=$ $\left(v_{k}, w_{k}\right)$. The condition $q_{k} \in U_{\varepsilon / 2}(a) \times U_{\varepsilon / 2}(s)$ means that $a_{k} \in U_{\varepsilon / 2}(a)$ and $s_{k} \in V_{\varepsilon / 2}(s)$. Consider a parameterization $q_{k}(\tau), 0 \leq \tau \leq 1$, of the segment $q_{k} q_{k+1} \subset \mathrm{h}_{s_{k}}$ with $q_{k}(0)=q_{k}, q_{k}(1)=q_{k+1}$. Then $q_{k}(\tau)=$ $\left(a_{k}(\tau), s_{k}\right)$ and the map $\tau \mapsto a_{k}(\tau) \in \mathrm{aY}$ is continuous. The assumption $q_{k+1} \notin U_{\varepsilon / 2}(a) \times U_{\varepsilon / 2}(s)$ would imply that there is $\tau^{\prime} \in(0,1]$ with $\max \left\{\left|x_{k} x_{k}\left(\tau^{\prime}\right)\right|_{w},\left|y_{k} y_{k}\left(\tau^{\prime}\right)\right|_{w}\right\}=\varepsilon / 2$, where $a_{k}(\tau)=\left(x_{k}(\tau), y_{k}(\tau)\right)$. Since still $q_{k}\left(\tau^{\prime}\right) \in U_{\varepsilon}(a) \times V_{\varepsilon}(s)$, we can apply Lemma 4.1 to conclude that

$$
\alpha \beta \leq \frac{\left|x_{k} x_{k}\left(\tau^{\prime}\right)\right|_{w}}{\left|y_{k} y_{k}\left(\tau^{\prime}\right)\right|_{w}} \leq \frac{1}{\alpha \beta}
$$

with $1>\beta \geq \sqrt{1-8 \varepsilon} \geq 1 / 2$, and

$$
\sinh \left(\left|q_{k} q_{k}\left(\tau^{\prime}\right)\right| / 2\right) \geq \frac{\alpha(1+\alpha) \sqrt{\alpha}}{8} \max \left\{\left|x_{k} x_{k}\left(\tau^{\prime}\right)\right|_{w},\left|y_{k} y_{k}\left(\tau^{\prime}\right)\right|_{w}\right\}
$$


Using that $\left|q_{k} q_{k}\left(\tau^{\prime}\right)\right| \leq|S|<t$, we see that

$$
\max \left\{\left|x_{k} x_{k}\left(\tau^{\prime}\right)\right|_{w},\left|y_{k} y_{k}\left(\tau^{\prime}\right)\right|_{w}\right\} \leq \frac{1}{2 c} \sinh (|S| / 2)<\varepsilon / 2
$$

in contradiction with the assumption. Thus $q_{k+1} \in U_{\varepsilon / 2}(a) \times V_{\varepsilon / 2}(s)$. Applying Lemma 4.1, we obtain

$$
\left|q_{k} q_{k+1}\right| \geq c \max \left\{\left|x_{k} x_{k+1}\right|_{w},\left|y_{k} y_{k+1}\right|_{w}\right\} .
$$

Arguments for even $k$ are similar with replacing $a \leftrightarrow s$ in Lemma 4.1,

Proposition 4.3. The distance $\delta$ on $\mathrm{Hm}$ is nondegenerate, if $\delta\left(q, q^{\prime}\right)=0$, then $q=q^{\prime}$ for any $q, q^{\prime} \in \mathrm{Hm}$.

Proof. Assume that $\delta\left(q, q^{\prime}\right)=0$ for some $q, q^{\prime} \in$ Hm. It means that for every $k \in \mathbb{N}$ there is a zz-path $S=S(k)$ between $q, q^{\prime}$ with $|S|<1 / k$.

Let $q=(a, s)$, where $a=(x, y), s=(v, w)$. We fix a sufficiently small $\varepsilon, 0<\varepsilon<1 / 16$, and take $t=t(\varepsilon)>0$ as in Lemma 4.2. Then for every $k \in \mathbb{N}, k \geq 1 / t$, the zz-path $S(k)$ satisfies the conclusion of Lemma 4.2. In particular, all vertices $q_{i}(k)$ of $S(k)$ lie in $U_{\varepsilon}(a) \times V_{\varepsilon}(s)$. Thus $q^{\prime}=q$ because $\varepsilon$ is taken arbitrarily.

\subsection{Completeness of the distance $\delta$}

Lemma 4.4. Given a harmonic 4-tuple $q=(a, s)$ with $a=(x, y), s=$ $(v, w)$, and $v^{\prime}$ lying on the open arc $d$ in $X$ determined by a that does not contain $w$, there is a harmonic $q^{\prime}=\left(a^{\prime}, s^{\prime}\right)$ with $s^{\prime}=\left(v^{\prime}, w\right)$, which is connected with $q$ by a zz-path $S$ with at most 3 sides. Moreover, if $s^{\prime} \in V_{\varepsilon}(s)$ for a sufficiently small $\varepsilon>0$, then $|S|<\gamma$ with $\gamma=\gamma(\varepsilon) \rightarrow 0$ as $\varepsilon \rightarrow 0$, where $V_{\varepsilon}(s)=\left\{\left(v^{\prime}, w^{\prime}\right) \in \mathrm{aY}:\left|v v^{\prime}\right|_{x}<\varepsilon,\left|w w^{\prime}\right|_{x}<\varepsilon\right\}$.

Proof. We construct a zz-path $S=\left\{\sigma_{i}\right\}, i=1,2,3$, as follows. We define $\sigma_{1}=s s_{1} \subset \mathrm{h}_{a}$ with $s_{1}=\left(v_{1}, w_{1}\right)$ so that $v_{a}^{\prime} \in \sigma_{1}$ (for the notation $v_{a}^{\prime}$ see sect. 3.1). Moreover, we can assume that $v_{a}^{\prime} \in \operatorname{int} \sigma_{1}$ unless $v^{\prime}=v$, in which case $s_{1}:=s$, and the construction of $S$ is finished with $\sigma_{1}=\sigma_{2}=\sigma_{3}=q$ degenerated. Then $\sigma_{1}$ is the segment on the line $\mathrm{h}_{a}$ between $q$ and $q_{1} \in \mathrm{Hm}$, $q_{1}=\left(a, s_{1}\right)$.

By construction, the pairs $s_{1}, s^{\prime}$ are in the strong causal relation and thus there is a (unique!) common perpendicular $a_{1}=\left(x_{1}, y_{1}\right)$ to them. We assume that $v_{1}$ lies on the arc $d$ in $X$, and $x_{1}$ lies on the arc in $X$ determined by $s$ that contains $x$.

Next, we define $\sigma_{2}:=a a_{1} \subset \mathrm{h}_{s_{1}}$ as the segment between $q_{1}$ and $q_{2}=$ $\left(a_{1}, s_{1}\right)$, and finally, we define $\sigma_{3} \subset \mathrm{h}_{a_{1}}$ as the segment $\sigma_{3}=\left(v_{1}\right)_{a_{1}} v_{a_{1}}^{\prime}=$ $\left(w_{1}\right)_{a_{1}} w_{a_{1}}$ between $q_{2}$ and $q_{3}=\left(a_{1}, s^{\prime}\right)=q^{\prime}$. In particular, $a^{\prime}=a_{1}$. Thus the segment $\sigma_{1} \subset \mathrm{h}_{a}$ uniquely determines our zz-path $S=\sigma_{1} \sigma_{2} \sigma_{3}$ connecting $q$ and $q^{\prime}$. 
To prove the last assertion of the lemma we parametrize the line $\mathrm{h}_{a} \subset$ $\mathrm{Hm}, h_{a}=\mathrm{h}_{a}(\tau), \tau \in \mathbb{R}$, so that $\mathrm{h}_{a}(0)=q, \mathrm{~h}_{a}(+\infty)=x, \mathrm{~h}_{a}(-\infty)=y$, and $\left|\mathrm{h}_{a}(\tau) \mathrm{h}_{a}\left(\tau^{\prime}\right)\right|=\left|\tau-\tau^{\prime}\right|$. The problem with the zz-path $S$ is that there is no constructive way to find the common perpendicular to given lines. We avoid this problem by defining a map $f: R_{+}^{2} \rightarrow d$ as follows. For $\tau \in \mathbb{R}$ we take the point $\mathrm{h}_{a}(\tau)=:\left(a, s_{1}\right)$ on the line $\mathrm{h}_{a}$. Next, we move along the line $\mathrm{h}_{s_{1}}$ by the distance $t \geq 0$ in the direction $v_{1} \in d$, where $s_{1}=\left(v_{1}, w_{1}\right)$. This gives us the point $\left(a_{1}, s_{1}\right)$ on the line $\mathrm{h}_{s_{1}}$. Finally, we move along the line $\mathrm{h}_{a_{1}}$ until we reach $\left(v^{\prime}, w\right) \in \mathrm{h}_{a_{1}}$ for some $v^{\prime} \in d$. Then we define $f(\tau, t):=v^{\prime}$. By the construction, $a_{1} \subset \bar{d}$ (the closure of the arc $d$ in $X$ ), thus the projection $w_{a_{1}} \in \mathrm{h}_{a_{1}}$ is well defined, hence the map $f$ is well defined.

Note that $f(0, t)=v=f(\tau, 0)$ for each $\tau \in \mathbb{R}, t \geq 0$, and that $f(\tau, t) \neq v$ for any $\tau \neq 0, t>0$, because otherwise we would have two different common perpendiculars to the distinct lines $\mathrm{h}_{s}, \mathrm{~h}_{s_{1}}$. The map $f$ is continuous which follows from uniqueness in Lemma 3.1. For $\gamma>0$ we define $v_{\gamma}^{ \pm}=f( \pm \gamma, \gamma)$, $\varepsilon_{\gamma}=\min \left\{\left|v v_{\gamma}^{ \pm}\right|_{x}\right\}$. Since the set

$$
A_{\gamma}=\{(\tau, t):-\gamma \leq \tau \leq \gamma, 0 \leq t \leq \gamma\} \subset \mathbb{R}_{+}^{2}
$$

is connected, for every $0 \leq \varepsilon \leq \varepsilon_{\gamma},\left(v^{\prime}, w\right) \in V_{\varepsilon}(s)$, there is $(\tau, t) \in A_{\gamma}$ such that $f(\tau, t)=v^{\prime}$. Now for any $0 \leq \varepsilon \leq \varepsilon_{1}$ and $\left(v^{\prime}, w\right) \in V_{\varepsilon}(s)$ we take the minimal $\gamma=\gamma(\varepsilon)$ such that $f(\tau, t)=v^{\prime}$ for $(\tau, t) \in A_{\gamma}$. We have $\left|\sigma_{1}\right|$, $\left|\sigma_{2}\right| \leq \gamma$, and clearly, $\gamma(\varepsilon) \rightarrow 0$ as $\varepsilon \rightarrow 0$.

Using Lemma 3.2, we find $\left|\sigma_{3}\right| \leq 2 \sqrt{\frac{\left|v_{1} v^{\prime}\right|\left|w w_{1}\right|}{\left|v_{1} w_{1}\right|\left|v^{\prime} w\right|}}$, where $v^{\prime}=f(\tau, t)$. Assuming that $\tau>0$, we normalize the metric of $X_{x}$ by the condition $|v w|_{x}=1$ (in the case $\tau<0$ we take $X_{y}$ instead of $X_{x}$ ). Then $\left|v_{1} w_{1}\right|_{x},\left|v^{\prime} w\right|_{x} \geq|v w|_{x}=$ 1. By Lemma 3.3

$$
\left|v v_{1}\right|_{x},\left|w w_{1}\right|_{x} \leq \frac{e^{\gamma}-1}{\alpha(1+\alpha)}|v w|_{x}=\frac{e^{\gamma}-1}{\alpha(1+\alpha)}
$$

By mononicity, $\left|v^{\prime} v_{1}\right|_{x} \leq\left|v v_{1}\right|_{x}$. Thus $\left|\sigma_{3}\right| \leq \frac{2\left(e^{\gamma}-1\right)}{\alpha(1+\alpha)} \leq c \gamma$ for some constant $c=c(\alpha)>0$ as $\varepsilon \rightarrow 0$. Hence $S=\left|\sigma_{1}\right|+\left|\sigma_{2}\right|+\left|\sigma_{3}\right| \leq(2+c) \gamma$, which completes the proof.

Proposition 4.5. The $\delta$-metric topology on $\mathrm{Hm}$ coincides with that induced from $X^{4}$.

Proof. Let $\tau$ be the topology on $\mathrm{Hm}$ induced from $X^{4}, \tau_{\delta}$ the $\delta$-metric topology. It follows from Lemma 4.2 that every $\tau$-open neighborhood $U_{\varepsilon}(a) \times$ $V_{\varepsilon}(s)$ of $q=(a, s) \in \mathrm{Hm}$ contains a $\delta$-metric ball $B_{t}(q)$ centered at $q$, where $t=t(\varepsilon)$. Hence, $\tau$ is not finer than $\tau_{\delta}$, i.e., every $\tau$-open set in $\mathrm{Hm}$ is $\tau_{\delta}$-open.

Conversely, given a $\delta$-metric ball $B_{t}(q)$ of radius $t>0$ centered at $q=$ $(a, s) \in \mathrm{Hm}$ we find a sufficiently small $\varepsilon>0$ such that every point $q^{\prime} \in$ $U_{\varepsilon}(a) \times V_{\varepsilon}(s), q^{\prime}=\left(a^{\prime}, s^{\prime}\right)$, is connected with $q$ by a zz-path of length $<t$. 
We find a short zz-path $S$ between $q$ and $q^{\prime}$ as follows. First, we take $q_{1}=\left(a_{1}, s_{1}\right) \in$ Hm with $s_{1}=\left(v^{\prime}, w\right) \in V_{\varepsilon}(s)$, where $s=(v, w), s^{\prime}=\left(v^{\prime}, w^{\prime}\right)$, and connect $q$ with $q_{1}$ by a zz-path $S_{1}$ as in Lemma 4.4 with $\left|S_{1}\right|<\gamma_{1}(\varepsilon)$. Applying the same construction, we find a zz-path $S_{2}$ between $q_{1}$ and $q_{2}=$ $\left(a_{2}, s^{\prime}\right)$ with $\left|S_{2}\right|<\gamma_{2}(\varepsilon)$. Then $s^{\prime}$ is the common perpendicular to $a^{\prime}, a_{2}$, and we connect $q_{2}$ with $q^{\prime}$ along $\mathrm{h}_{s^{\prime}}$.

Recalling the construction of Lemma 4.4, we see that the width of the strip between $a$ and $a_{1}$ is at most $\gamma_{1}(\varepsilon)$, and the width of the strip between $a_{1}$ and $a_{2}$ is at most $\gamma_{2}(\varepsilon)$. Combining with Lemma 3.3. this allows to conclude that $a_{2} \in U_{\gamma_{3}(\varepsilon)}(a)$ for some $\gamma_{3}(\varepsilon)$ with $\gamma_{3}(\varepsilon) \rightarrow 0$ as $\varepsilon \rightarrow 0$. By Lemma 3.2, we find that $\left|q_{2} q^{\prime}\right| \leq \gamma_{4}(\varepsilon)$ with $\gamma_{4}(\varepsilon) \rightarrow 0$ as $\varepsilon \rightarrow 0$. It follows that $\delta\left(q, q^{\prime}\right) \leq \gamma(\varepsilon)$ for some $\gamma(\varepsilon)$ with $\gamma(\varepsilon) \rightarrow 0$ as $\varepsilon \rightarrow 0$. Taking $\varepsilon>0$ sufficiently small, we find that any $q^{\prime} \in U_{\varepsilon}(a) \times V_{\varepsilon}(s)$ lies in $B_{t}(q)$. Hence $\tau=\tau_{\delta}$.

Proposition 4.6. The distance $\delta$ on $\mathrm{Hm}$ is complete.

Proof. Let $\left\{q_{i}\right\} \in \mathrm{Hm}$ be a Cauchy sequence with respect to the metric $\delta$. For a given $t>0$ we have $\delta\left(q_{i}, q_{j}\right)<t$ for all sufficiently large $i, j$. By approximation, we find for each $i, j$ a zz-path $S_{i j}$ between $q_{i}, q_{j}$ with $\left|S_{i j}\right| \leq 2 \delta\left(q_{i}, q_{j}\right)$. Then for all sufficiently large $i, j$ we have $\delta\left(q_{i}, q_{j}\right)<t / 2$ and thus $\left|S_{i j}\right|<t$.

Now, fix $\varepsilon>0, \varepsilon \leq 1 / 16$, and apply Lemma 4.2 to the zz-paths $S_{i j}$ for a fixed $i$ and all sufficiently large $j$. Then $q_{j} \in U_{\varepsilon}\left(a_{i}\right) \times V_{\varepsilon}\left(s_{i}\right)$, where $q_{i}=$ $\left(a_{i}, s_{i}\right)$. Moreover, the estimates of Lemma 4.2 (and the triange inequality) imply that the sequences $x_{j}, y_{j} \in X_{w}, v_{j}, w_{j} \in X_{x}$ are Cauchy with respect to the metrics $X_{w}, X_{x}$, where $a_{j}=\left(x_{j}, y_{j}\right), s_{j}=\left(v_{j}, w_{j}\right)$. By Corollary 2.5. there are $\operatorname{limits} x=\lim x_{j}, y=\lim y_{j}, v=\lim v_{j}, w=\lim w_{j}$, and the 4-tuple $q=(x, y, v, w)$ is harmonic. By Proposition 4.5, $\delta\left(q, q_{j}\right) \rightarrow 0$.

Proof of Theorem 1.1. It follows from Propositions 4.3, 4.5 and 4.6 that the distance $\delta$ on $\mathrm{Hm}$ is nondegenerate, that the $\delta$-metric topology coincides with that induced from $X^{4}$, in particual, the metric space $(\mathrm{Hm}, \delta)$ is locally compact, and that $(\mathrm{Hm}, \delta)$ is complete. By definition, $(\mathrm{Hm}, \delta)$ is a length space. By Hopf-Rinov theorem, $(\mathrm{Hm}, \delta)$ is proper, that is, closed balls are compact. A standard argument shows that $(\mathrm{Hm}, \delta)$ is geodesic, i.e., for any two points $p, q$ with $\delta(p, q)<\infty$ there is a geodesic $p q$ between them.

\section{References}

[Bu17] S. Buyalo, Möbius structures and timed causal spaces on the circle, Algebra i analys, 29:5 (2017), 1-50, translation in St. Petersburg Math. J. 29 (2018), no. 5, 715-747. 
[Bu18] S. Buyalo, On the inverse problem of Möbius geometry on the circle, arXiv: math. MG/1810.03133.

[DDDL] A. Daniilidis, G. David, E. Durand-Cartagena, and A. Lemenant, Rectifiability of self-contracted curves in the Euclidean space and applications, The Journal of Geometric Analysis, 25(2):1211-1239, Apr 2015.

[DDDR] A. Daniilidis, R. Deville, E. Durand-Cartagena, and L. Rifford, Self-contracted curves in Riemannian manifolds, J. Math. Anal. Appl., 457:1333-1352, 2018.

[DLS] A. Daniilidis, O. Ley, and S. Sabourau, Asymptotic behaviour of self-contracted planar curves and gradient orbits of convex functions, Journal de Mathmatiques Pures et Appliques, 94(2):183 199, 2010.

[FS12] T. Foertsch, V. Schroeder, Ptolemy circles and Ptolemy segments. Arch. Math. (Basel) 98 (2012), no. 6, 571581.

[FS13] T. Foertsch, V. Schroeder, Metric Möbius geometry and a characterization of spheres, Manuscripta Math. 140 (2013), no. 3-4, 613-620.

[Le] A. Lemenant, Rectifiability of non Euclidean planar self-contracted curves, Confluentes Mathematici, 2017.

[Ohta] Shin-ichi Ohta, Self-contracted curves in CAT(0)-spaces and their rectifiability, mathArXiv:1711.09284, 2017

[LOZ] N. Lebedeva, Shin-ichi Ohta, V. Zolotov, Self-contracted curves in spaces with weak lower curvature bound, mathArXiv:1902.01594.

[ST] E. Stepanov and Y. Teplitskaya, Selfcontracted curves have finite length, Journal of the London Mathematical Society, 96(2):455481.

[Zo18] V. Zolotov, Subsets with small angles in self-contraced curves, arXiv:1804.00234 [math.MG], 2018. 